\title{
II Ökonomische Charakteristika von Wohnungsmärkten und korrelierender Regulierungsbedarf
}

\section{Wesensmerkmale von Wohnungsmärkten}

Für die politische Dimension der Diskussion um Wohnungsmärkte ist von Bedeutung, dass es sich beim Wohnen um ein zentrales Grundbedürfnis des Menschen handelt, für das die Haushalte auch mit mittleren Einkommen einen signifikanten Anteil des zur Verfügung stehenden Geldes ausgeben, und zwar je nach Höhe des Gesamthaushaltsnettoeinkommens zwischen der knappen Hälfte (46,7 \% bei einem geringen Einkommen unter $1.300 €$ pro Monat) und einem guten bzw. knappen Drittel bei einem monatlichen Einkommen zwischen 2.600 bis $3.600 €(37,4 \%)$ bzw. 3.600 bis $5.000 €(34,7 \%) .{ }^{27}$ Schon deshalb sind Preissteigerungen von ungleich höherer Relevanz und politischer Brisanz als solche in anderen Märkten, die keine vergleichbare Haushaltsrelevanz aufweisen. ${ }^{28}$

Dies erklärt die hohe soziale Sensibilität des Themas. Für das weitere Verständnis des Immobilienmarktes sind sodann jedoch andere Besonderheiten von Bedeutung. So weisen Wohnungen eine lange Lebensdauer auf - anders als etwa Autos oder gar Lebensmittel wie Brot, die ebenfalls Grundbedürfnisse auf Mobilität und Ernährung abdecken. In der Konsequenz kann das Angebot nicht mit gleicher Geschwindigkeit wie etwa im Brotmarkt der Nachfrage angepasst werden. Planerische Fehlprognosen - etwa über den (un-)erwarteten Zuzug in eine Stadt wie Berlin - haben deshalb ungleich größere Auswirkungen. Ferner dominiert vor diesem Hintergrund der Bestandsmarkt den Wohnungsmarkt. Der Markt für neue Wohnungen ist aufgrund einer Bereitstellungsquote von inzwischen

27 Siehe zu den Zahlen für das Jahr 2016 Statistische Bundesamt, Datenreport 2018, S. 202, abrufbar im WWW unter der URL https://www.destatis.de/DE/Service/Statistik-Campus/Datenre port/Downloads/datenreport-2018.pdf;jsessionid=CA944B28B0BD420B2E79E058B5 A2 A200.in ternet742?_blob=publicationFile (zuletzt abgerufen am 1.7.2020).

28 Siehe etwa die stark gestiegenen Strompreise, bei denen gleichwohl eine Diskussion über deren Bezahlbarkeit unter dem Stichwort der „Energiearmut“ geführt wird; dazu Buckler Sozialtarife für Stromverbraucher: Das französische System als Vorbild? EU- und (finanz-)verfassungsrechtliche Vorgaben für den Schutz einkommensschwacher Stromverbraucher in Deutschland, EnWZ 2013, 114; Schlack Energiearmut - Herausforderung in Zeiten der Energiewende. Entlastungsmöglichkeiten für einkommensschwache Letztverbraucher, EnWZ 2013, 27.

Ә OpenAccess. ( 2020 Juergen Kuehling, publiziert von De Gruyter. (cc) BY-NC-ND Dieses Werk ist lizenziert unter der Creative Commons Attribution-NonCommercial-NoDerivatives 4.0 License. 
deutlich weniger als 1\% pro Jahr dagegen von deutlich geringerer Relevanz. ${ }^{29}$ In regulatorischer Perspektive hat dies erhebliche Folgen: Hoheitliche Interventionen wirken nur mit starker Verzögerung und umgekehrt können angesichts der Langfristigkeit von Immobilieninvestitionen Investoren nur „träge“ reagieren, zumal auch die Realisierungszeiten von Immobilienprojekten vergleichsweise lang sind. Schon daraus folgt, dass Stabilität und Berechenbarkeit der wohnungspolitischen Regulierung von großer Bedeutung sind. Die Langlebigkeit von Immobilien führt im Übrigen dazu, dass auch die Vertragsbeziehungen - insbesondere zwischen Vermieter und Mieter - regelmäßig langfristiger Natur sind. So vermieten private Einzelvermieter ihre Wohnung durchschnittlich für fast zehn Jahre. In etwa die Hälfte der Mietverträge läuft länger als fünf Jahre, länger als zehn Jahre immerhin noch ein Drittel und fast ein Achtel der Verträge sogar länger als 20 Jahre. ${ }^{30}$

Der immobile Charakter des Gutes bedingt ferner, dass geografische Ausweichreaktionen der Nachfrager nur beschränkt möglich sind. So gibt es sicherlich größere Leerstände in manchen Regionen Deutschlands. ${ }^{31}$ Diese lassen sich jedoch nicht in die „Schwarmstädte“ verlagern. Insofern steht die Politik oftmals in ein- und demselben Bundesland vor zwei gegenläufigen und gleichermaßen fordernden Entwicklungen, nämlich der wachsenden Bevölkerung in wenigen urbanen Regionen und der schrumpfenden Bevölkerung auf dem Land. ${ }^{32}$ Interessant ist dabei, dass in jüngerer Zeit die enge Korrelation $\mathrm{zu}$ den regionalen

29 Siehe dazu allgemein Eekhoff Wohnungs- und Bodenmarkt, 2. Aufl. 2006, S. 3 f.; siehe ferner zur weiteren Entwicklung der Neubauquote (Neugebaute Wohnungen in Relation zu dem bisherigen Wohnungsbestand): 0,58\% (2016), 0,59\% (2017) und 0,61\% (2018), vgl. zu den jährlich fertiggestellten Wohnungen Statistisches Bundesamt (Hrsg.), Bauen und Wohnen, 2019, S. 42 und 66, abrufbar im WWW unter der URL https://www.destatis.de/DE/Themen/Branchen-Unterneh men/Bauen/Publikationen/Downloads-Bautaetigkeit/baufertigstellungen-bauherren-pdf5311201.pdf?_blob=publicationFile (zuletzt abgerufen am 1.7.2020), und für den jährlichen Wohnungsgesamtbestand dass., Bautätigkeit und Wohnungen, 2019, S. 6, abrufbar im WWW unter der URL https://www.destatis.de/DE/Themen/Gesellschaft-Umwelt/Wohnen/Publikatio nen/Downloads-Wohnen/bestand-wohnungen-2050300187004.pdf?_blob=publicationFile (zuletzt abgerufen am 1.7.2020).

30 Haus \& Grund, Vermieterbefragung, Ergebnisse 2019, S. 4, abrufbar im WWW unter der URL https://www.hausundgrund.de/sites/default/files/downloads/vermieterbefragungdeutsch land2019.pdf (zuletzt abgerufen am 1.7.2020).

31 Sachsen-Anhalt (12,6\%), Sachsen (10,9\%) und Thüringen (10,0\%) wiesen 2017 die höchsten Leerstandsquoten auf, vgl. BBSR, Schwerpunktthema Leerstandsentwicklung im Stadtumbau, 2020, abrufbar im WWW unter der URL https://www.staedtebaufoerderung.info/StBauF/Shared Docs/Publikationen/StBauF/Stadtumbau/Schwerpunkthtema5.pdf?_blob=publicationFile\&v=5 (zuletzt abgerufen am 1.7.2020).

32 BBSR, Wohnungs- und Immobilienmärkte 2016, Analysen Bau.Stadt.Raum, Bd. 12, 2016, S. 6. 
Arbeitsmärkten aufgrund der insgesamt sehr guten Entwicklung der Arbeitsmärkte in Deutschland partiell entkoppelt worden ist. ${ }^{33}$ Gleichwohl verbleiben regionale Ungleichgewichte. Diesen kann durch politische Interventionen - wie etwa die Verlagerung von Behörden oder die Ansiedlungen von Hochschulen bzw. Forschungs- und Lehrabteilungen in strukturschwache Regionen - nur sehr begrenzt entgegengesteuert werden. Hier zeigt sich auch die Abhängigkeit des nachgelagerten Wohnungsraummarktes vom vorgelagerten Bodenmarkt, der ein physisch begrenztes, nicht vermehrbares knappes Gut darstellt. ${ }^{34}$ Vermehrbar ist daher nur die Dichte der Bebauung auf einer gegebenen Fläche und dies auch nur begrenzt.

Die Bedeutung des Standortes führt jedoch letztlich zur Notwendigkeit einer noch kleinteiligeren Betrachtung und in der Konsequenz - neben den gebäudeinternen qualitativen Kriterien wie beispielsweise die Wohnungsgröße, die Raumanzahl oder der Bauzustand - zur Feststellung der Heterogenität des Wirtschaftsgutes Wohnung. Jede einzelne Wohnung zeichnet sich durch so viele Besonderheiten aus (Lage, Schnitt, Ausstattung, Belichtung, Beschallung etc.), dass sie nur begrenzt substituierbar ist durch eine andere. ${ }^{35}$ Angesichts der geringen Homogenität ist auch die Markttransparenz geringer als etwa bei einem homogenen Wirtschaftsgut wie Autos. Schließlich ist ein hoher Kapitaleinsatz erforderlich und der Transfer von Wohnungen ist mit hohen Transaktionskosten verbunden, was wiederum die Fungibilität des Gutes reduziert. ${ }^{36}$ Aus Sicht des Nachfragers tritt noch die weitgehend fehlende Teilbarkeit des Konsums hinzu: So kann der Bewohner einer Immobilie bei steigenden Quadratmeterpreisen nicht kurzfristig mit einer Verringerung der spezifischen Nachfrage reagieren, indem er etwa auf einen Teil der Fläche verzichtet („Untermieter“). ${ }^{37}$

33 Simons/Weiden Schwarmverhalten, Reurbanisierung und Suburbanisierung, Information zur Raumentwicklung, BBSR Heft 3.2016, S. 265f., abrufbar im WWW unter der URL https://d-nb.info/ 1155025490/34 (zuletzt abgerufen am 1.7.2020).

34 Zur Abhängigkeit Eekhoff Wohnungs- und Bodenmarkt, 2. Aufl. 2006, S. 17 f.; hinsichtlich der Auswirkung hoher Boden- auf Wohnungspreise im Zusammenspiel mit anderen Faktoren (wie dem spekulativen Zurückhalten von Baugenehmigungen) BBSR, Bauland als Engpassfaktor für mehr bezahlbaren Wohnraum, 2017, passim und S. 15; BBSR, Wohnungs- und Immobilienmärkte 2016, Analysen Bau.Stadt.Raum, Bd. 12, 2016, S. 88.

35 Gondring Immobilienwirtschaft, 3. Aufl. 2013, S. $16 \mathrm{f}$.

$36 \mathrm{Zu}$ diesen Aspekten wiederum Gondring aaO, S. 17.

37 Schuldt Mietpreisbremse. Eine juristische und ökonomische Untersuchung der Preisregulierung für preisfreien Wohnraum, 2017, S. 52. 


\section{Marktversagen und Notwendigkeit staatlicher Eingriffe?}

Vor dem Hintergrund dieser ökonomischen Besonderheiten stellt sich die Frage nach der prinzipiellen oder - mit Blick auf die Marktentwicklungen - auch nur punktuellen Notwendigkeit staatlicher Eingriffe, da der Markt seine Aufgaben nicht richtig erfüllen kann und es also zu einem Marktversagen kommt.

\section{a) Fälle eines Marktversagens und prinzipielle Relevanz in Wohnungsmärkten}

Auch wenn die Theorie des Marktversagens teils grundsätzlich ${ }^{38}$, teils in den Details $^{39}$ in Frage gestellt wird, liefert sie doch einen sehr hilfreichen Rahmen für die Analyse hoheitlicher Interventionen in Märkte und die Bewertung ihres Beitrags zur tatsächlichen oder nur vermeintlichen Lösung tatsächlicher oder nur vermeintlicher Defizite des Marktgeschehens.

Ein Marktversagen liegt vor, wenn die Verteilung der Güter über den Markt nicht zu einer pareto-effizienten Allokation führt, d. h. ein Zustand, in dem es nicht möglich ist, eine Zieleigenschaft zu verbessern, ohne eine andere negativ zu beeinflussen. ${ }^{40}$ Es wird eine Reihe von nachgerade „klassischen“ Gründen für ein solchermaßen verstandenes Marktversagen angeführt.

So bestehen Informationsasymmetrien bzw. weiter gefasst Transparenzdefizite, da den Anbietern oftmals nicht hinreichend verlässliche Daten über die künftige Nachfrage vorliegen und den Nachfragern regelmäßig angesichts der Heterogenität des Angebots kein verlässlicher Überblick zur Verfügung steht. Die Beseitigung dieser Transparenzdefizite ist nur schwer möglich und jedenfalls mit erheblichen Transaktionskosten verbunden. ${ }^{41}$ Die Digitalisierung erleichtert hier allerdings den Zugriff auf Informationen. Aus der Perspektive des einzelnen Mieters führen die fehlende Teilbarkeit und die spezifischen Investitionen in eine Wohnung (insbesondere auch in das soziale Umfeld) sowie die hohen Transaktionskosten eines Wohnungswechsels zu „Lock-in“-Effekten, die ein Marktversa-

38 Siehe die Auseinandersetzung mit verschiedenen kritischen Ansätzen gegenüber der paretianischen Wohlfahrtsökonomik und dem einer Marktversagensanalyse zugrundeliegenden Modell der vollständigen Konkurrenz etwa bei Fritsch Marktversagen und Wirtschaftspolitik, 10. Aufl. 2018, S. 57 ff.; zur allgemeinen Kritik auch Schuldt Mietpreisbremse. Eine juristische und ökonomische Untersuchung der Preisregulierung für preisfreien Wohnraum, 2017, S. 94.

39 Z.B. Schuldt Mietpreisbremse. Eine juristische und ökonomische Untersuchung der Preisregulierung für preisfreien Wohnraum, 2017, S. $96 \mathrm{ff}$.

40 Vgl. zum Begriff Mayer Theorie und Politik des Wohnungsmarktes, 1998, S. 81.

41 Ebenda, S. 53. 
gen in Form einer Informationsasymmetrie begründen können. Der Mieter wird eher - als bei anderen Gütern - bereit sein, am individuellen Anbieter festzuhalten und eine Mieterhöhung zu tragen als die Wohnung zu wechseln. Bei Vertragsschluss kann er aber ein etwaiges opportunistisches Verhalten in Form späterer hoher Mietanpassungsforderungen des Vermieters nicht sicher abschätzen und auch nicht ausschließen. In diesem Zusammenhang zeigt sich auch, dass der Mieter in einem großen Umfang spezifische Investitionen in seine Wohnung und vor allem sein Mikroumfeld tätigt, was zu hohen „Lock-in“-Effekten führt. Im Zweifel möchte der Mieter am liebsten in der angestammten Wohnung, jedenfalls aber im angestammten Mikrobereich wohnen bleiben, da er sich mit diesem identifiziert und dort verwurzelt ist.

Die eingangs dargestellte lange Bereitstellungsdauer von mehr Wohnraum und die Dominanz des Bestandsmarktes führen zu einem jedenfalls kurzfristig preisunelastischen Angebot und nur einer mittel- bis langfristigen Preiselastizität von Angebot und Nachfrage..$^{42}$ Im Fall der Schwarmstädte bedeutet dies, dass ein starker Nachfrageüberhang nur mit erheblichem zeitlichem Verzug zur Ausweitung des Angebots führt. In der Konsequenz steigen die Preise in dieser Anpassungsphase deutlich. Hinzu kommt, dass im Falle der Ausschöpfung knapper Bodenreserven (und von Nachverdichtungsmöglichkeiten) das Ausmaß der Angebotserweiterung limitiert ist. Folglich werden bei unverminderter Nachfrage die Preise steigen, bis die Zahlungsbereitschaft abgeschöpft ist und in der Folge die Nachfrage nachlässt und sich gegebenenfalls in andere Räume verlagert.

Der Vollständigkeit halber sei darauf hingewiesen, dass auf Wohnungsmärkten auch externe Effekte insofern auftreten als der Wert einer Wohnung stark durch die Entwicklung der näheren Umgebung geprägt wird. Dies ist etwa positiv durch die Verbesserung der verkehrlichen Anbindung oder die Schaffung von nahegelegenen Grün- und Naherholungsflächen der Fall bzw. negativ durch die Änderung einer Flugroute mit gesteigertem Verkehrslärm im Mietobjekt.

Liegen diese Gründe eines Marktversagens vor, ist aus ordnungspolitischer Sicht eine staatliche Intervention gerechtfertigt, wenn diese zu einer Verbesserung der Situation, also zu einer effizienteren Güterverteilung, beiträgt. Dabei ist zu beachten, dass staatliches Handeln seinerseits fehleranfällig ist und Transaktionskosten generiert und daher gegebenenfalls trotz bestehender marktlicher Defizite zu einer Verschlechterung der Situation führt.

Diese potenziellen Marktversagensgründe sind strikt zu trennen von einem distributiven „Marktversagen“, d.h. also der Einschätzung eines „ungerechten“ Verteilungsergebnisses durch den Markt. Da der Markt auf Preissignale und nicht

42 Vgl. ebenda, S. 53. 
auf spezifische Gerechtigkeitsvorstellungen reagiert, kann der Markt so verstandene Gerechtigkeitsziele von vornherein nicht erreichen. So kann der Markt etwa das von Kevin Kühnert in seiner damaligen Funktion als Vorsitzender der Jusos ausgegebene Ziel, dass ,jeder maximal den Wohnraum besitzen (sollte), in dem er selbst wohnt“, nicht erreichen. ${ }^{43}$ Insoweit ist lediglich eine Umverteilung möglich. Im „Modell Kühnert“ wäre diese maximal radikal.

\section{b) Unklarheit über Regulierungsziele bei hoheitlichen Maßnahmen}

Die zur Rechtfertigung von Interventionen angeführten Gründe in Gesetzesänderungen oder entsprechenden Vorschlägen lassen allzu oft keine klare Zuordnung der Maßnahmen anhand dieser Marktversagensanalyse erkennen bzw. weisen jedenfalls nicht differenziert auf die Probleme auf dem Wohnungsmarkt hin. Stattdessen wird einfach mit dem Befund steigender Mieten oder der Beobachtung von Gentrifizierungsprozessen argumentiert. ${ }^{44}$ Für die grundrechtliche Bewertung interessant ist auch die Beobachtung, dass die Gemeinwohlziele regelmäßig nicht sorgfältig genug genannt werden. So wird meist sehr pauschal auf die Schwierigkeiten einkommensschwacher Haushalte und vor allem von Familien, geeigneten Wohnraum in begehrten Lagen zu erlangen, verwiesen, ohne dass näher auf eine diesbezügliche Ausrichtung der Steuerungsinstrumente eingegangen wird. Erst recht wird nicht klar genug herausgearbeitet, inwiefern die vorgeschlagene Maßnahme zu einer Zweckerreichung beiträgt, welche negativen Effekte $\mathrm{zu}$ befürchten sind und inwiefern die Eingriffe gleichwohl aus überwiegenden Gemeinwohlgründen gerechtfertigt werden können. So wird etwa diffus auf die Vermeidung einer Gentrifizierung ${ }^{45}$ verwiesen, ohne dass klar wird, welche

43 Siehe dazu etwa den Bericht abrufbar im WWW unter der URL https://www.stuttgarter-zei tung.de/inhalt.kevin-kuehnert-will-immobilienbesitz-beschraenken-jeder-sollt-maximal-denwohnraum-besitzen-in-dem-er-selbst-wohnt.95cbac2a-261a-4c24-b74a-360d03c969e7.html (zuletzt abgerufen am 1.7.2020).

44 Siehe für die Einführung der „Mietpreisbremse“ den Gesetzesentwurf v. 10.11. 2014, BT-Drs. 18/ 3121, S. $11 \mathrm{f}$. und 15, wo letztlich nur pauschal auf Gentrifizierungsprozesse, steigende Mieten und das Nahziel der Dämpfung der Wiedervermietungsmieten verwiesen und zugleich eingeräumt wird, dass die „Mietpreisbremse“ „keinen zusätzlichen Wohnraum“ generiert; bei Einführung der Möglichkeit einer Herabsetzung der Kappungsgrenze auf 15\% wird ebenso wenig näher auf Regulierungsziele eingegangen, sondern lediglich auf das Nahziel der Dämpfung der Mietpreissteigerungen verwiesen, vgl. hierzu Beschlussempfehlung zum Gesetzesentwurf v. 12.12. 2012, BTDrs. 17/11894, S. 21 u. 23. Nähere Hinweise auf volkswirtschaftliche oder empirische Untersuchungen zu den eingeführten Maßnahmen fehlen.

45 Entwurf zum Mietrechtsnovellierungsgesetz v. 10.11.2014, BT-Drs. 18/3121, S. 11 und 19. 
dahinterstehenden Gemeinwohlziele im Fall der erfolgreichen Bekämpfung jenes Prozesses verfolgt und auch mit hinreichender Wahrscheinlichkeit erreicht werden können. Angesichts der aufgezeigten Besonderheiten einer hohen Identifikation des Mieters mit seinem Mikroumfeld liegt wohl ein „Recht auf Heimat“46 nahe, das auf den ersten Blick allemal sympathisch und auch politisch nachvollziehbar erscheint. Es wirft jedoch Definitionsschwierigkeiten auf: Für wen gilt es und für wen muss es geschützt werden? Für die noch einfacher zu identifizierende Gruppe der Geringverdiener bzw. für Familien? Oder etwa für die schwer eingrenzbare Gruppe der „Ortsansässigen“? Denn womit qualifiziert man sich als Ortsansässiger? Vermutlich am ehesten durch eine eigene lange Lebensdauer im Viertel (oder etwa die der Eltern?). Das kollidiert jedoch mit dem - auch unionsrechtlich - fundierten Gedanken der Freizügigkeit (Art. 11 GG; Art. 21, 45 AEUV). Inwiefern muss sodann ein Beruf hinzukommen, der vor Ort auszuüben ist, wie es etwa anklingt, wenn dafür plädiert wird, dass Berufsgruppen wie Polizisten oder Lehrer arbeitsortsnah bezahlbaren Wohnraum finden müssen? Dabei ist auch darauf hinzuweisen, dass teils der Schutz vor Gentrifizierung in Vierteln gefordert und gefördert wird, in denen es ohnehin bereits in erheblichem Umfang zu einem Austausch der Bevölkerung gekommen ist. Das gilt etwa für den Prenzlauer Berg in Berlin, in dem der Anteil der „Vorwende-Bewohner“ bzw. entsprechender sozialer Milieus, die noch immer im Prenzlauer Berg leben, überschaubar sein dürfte. Es wäre jedenfalls einer vertieften Untersuchung wert, inwiefern hier „Gentrifizierer der ersten Generation“ gegen solche der zweiten oder dritten Generation geschützt werden. ${ }^{47}$ Oder geht es um ein ganz anderes Ziel, nämlich um eine gewünschte Heterogenität der Wohnbevölkerung? Wenn ja, warum sollte das notwendig sein? Aus Sicherheitsgründen? Diese entstehen aber

46 Für die Anerkennung eines solchen Rechts im Zusammenhang mit Umsiedlungsmaßnahmen zugunsten des Braunkohletagebaus Baer NVwZ 1997, S. 27 ff., die Heimat definiert als „freiwillig gewählter, identitätsstiftender, soziokultureller, territorial bezogener und gesicherter Lebenszusammenhang“ und ergänzt: „Die Größe der Heimat kann im Einzelfall differieren, wird aber regelmäßig mit der einer Gemeinde zusammenfallen und über die einzelne Wohnung hinausgehen.“ Das BVerfG hat ein eigenständiges „Recht auf Heimat“ aus Art. 11 GG in diesem Zusammenhang allerdings abgelehnt und entsprechende Schutzwirkungen aus der Eigentumsfreiheit des Art. 14 GG abgeleitet, siehe BVerfG, Urt. v. 17.12.2013-1 BvR 3139/08, Rn. 263 ff., abrufbar im WWW unter der URL http://www.bverfg.de/e/rs20131217_1bvr313908.html - ECLI:DE: BVerfG:2013:rs20131217.1bvr313908 (zuletzt abgerufen am 1.7.2020).

47 Empfehlenswert in literarischer Hinsicht insoweit das Buch von Schulz Skandinavisches Viertel, 2018, das von den Umbrüchen in diesem Teil des Prenzlauer Bergs aus der Perspektive eines selbst erklärten „Anti-Gentrifizierungs-Makler“ erzählt, der es sich zur Aufgabe gemacht hat, gleichsam als „Türsteher“ unpassenden Neureichen den Zuzug in „sein Viertel“ zu verwehren. 
doch eher in Problemvierteln und nicht in gentrifizierten Gegenden. ${ }^{48}$ Ist Heterogenität gegebenenfalls ein öffentliches Gut oder nur ein schlicht politisch gewünschtes Ziel? Sorgt Heterogenität gar, wie teilweise behauptet wird, für eine Kreativität und Produktivität ${ }^{49}$ ? Gibt es dafür einen belastbaren empirischen Beleg?

Letztlich geht es demnach um das gesetzte Ziel, dass der Wohnraum bezahlbar bleiben soll. Aber wie viel Wohnraum wo und für wen genau? Denn das Problem, dass sich in jenen angespannten Wohnungsmärkten abspielt, lässt sich ja weder leugnen, noch wegdefinieren: Es wollen dort einfach mehr Menschen leben als Wohnraum vorhanden ist. Kurzum: Wohnraum ist knapp. Dann gibt es aber ganz grundsätzlich nur die Möglichkeit, den vorhandenen Wohnraum zu erweitern (Nachverdichtung etc.) oder die Nachfrage zu drosseln. Letzteres kann wiederum dadurch erfolgen, dass die Nachfrage „umgelenkt“ wird und insbesondere die Ansiedlung jenseits der angespannten Wohnungsmärkte attraktiver wird - etwa durch den ÖPNV-Anschluss von Umlandgemeinden. Andernfalls bleibt nur die Möglichkeit, dass die Menschen in angespannten Wohnungsmärkten ihren durchschnittlichen Wohnbedarf einschränken. Erfolgt aber weder ein Ausbau noch eine Drosselung der Nachfrage, verschärft sich der (verteilungspolitische) Kampf um den knappen Wohnraum. Drosselt der Gesetzgeber jetzt noch das Preissignal, indem etwa durch Kappungen der Mieten derselbe vorhandene Wohnraum „günstiger“ wird, steigt die Nachfrage weiter, da mehr Personen sich mehr von diesem vergünstigten Wohnraum leisten können. Zugleich wird das Angebot im Ergebnis verknappt. Denn selbst wenn angesichts des unregulierten Neubausegments dort die Preise in die Höhe schießen und dadurch zusätzliche Investitionsanreize entstehen, ist dieser Effekt doch geringer als die gegenläufigen Effekte. So führen derart radikale und auch überraschende Markteingriffe wie in Berlin zur Verunsicherung von Investoren: Wenn auch Neubauten gegenwärtig von der Regulierung freigestellt sind, wer garantiert, dass sich dies nach einem weiteren Politikschwenk nicht für die getätigten Investitionen in einem investitionsrelevanten Zeitraum ändert? Hier zeigen sich die negativen Effekte einer disruptiven Regulierungspolitik in einem auf langfristiges Vertrauen angelegten Investitionsumfeld. Noch viel deutlicher sind jedoch bereits jetzt die Effekte der Angebotsverknappung durch die Verlagerung des Mietwohnmarktes in den Verkaufswohnmarkt. So zeigt sich schon binnen kurzer Zeit

48 Siehe dazu Hinweise bei Weigelt Die wachsende Stadt als Herausforderung für das Recht. Rechtliche Instrumente zum Erhalt und zur Schaffung heterogener Bevölkerungsstrukturen in der Innenstadt, 2016, S. $16 \mathrm{f}$.

49 Dazu Weigelt aaO, S. 17, allerdings ohne, dass insoweit wirklich belastbare empirische oder andere Studien ersichtlich sind, die das hinreichend belegen. 
als offensichtlicher Effekt des Berliner „Mietendeckels“, dass im verschärft regulierten Marktsegment der Verkauf der bislang als Mietobjekte gehaltenen Wohnungen mit Baujahr älter als 2014 ansteigt. ${ }^{50}$ Insoweit wird der (verteilungspolitische) Kampf nochmals verschärft. Schon jetzt sei auf die massiv kontraproduktiven sozialen Effekte verwiesen, da im Kaufsegment sowie im deutlich reduzierten Neuvermietungssegment gerade einkommensschwache Haushalte besonders benachteiligt sind.

50 Siehe die Nachweise, dass bezogen auf den Zeitraum des Stichtags der Berliner Regelung, ab dem die Mieten „eingefroren“ wurden (18.6.2019), ein Anstieg der Verkaufsangebote im Marktsegment der verschärft regulierten Mietobjekte mit Baujahr älter als 2014 um 44\% (!) erfolgte, abrufbar im WWW unter der URL https://embed.presseportal.de/de/31321/article/4625790 (zuletzt abgerufen am 1.7.2020). 